\title{
Superconducting Tunnel Junction Array Development for High-Resolution Energy-Dispersive X-ray Spectroscopy
}

\author{
S. Friedrich, ${ }^{1,3 \star}$ C.A. Mears, ${ }^{1}$ B. Nideröst, ${ }^{1}$ L.J. Hiller, ${ }^{1}$ M. Frank, ${ }^{1}$ S.E. Labov, ${ }^{1}$ A.T. Barfknecht, ${ }^{2}$ \\ and S.P. Cramer ${ }^{3}$ \\ ${ }^{1}$ Physics and Space Technology Directorate, Lawrence Livermore National Laboratory, P.O. Box 808, L-418, \\ Livermore, CA 94550 \\ ${ }^{2}$ Conductus Inc., 969 West Maude Avenue, Sunnyvale, CA 94086 \\ ${ }^{3}$ Physical Biosciences Division, Lawrence Berkeley National Laboratory, MS 6-2100, Berkeley, CA 94720
}

\begin{abstract}
Cryogenic energy-dispersive X-ray detectors are being developed because of their superior energy resolution (10 eV FWHM for keV X-rays) compared to that achieved in semiconductor energy-dispersive spectrometry (EDS) systems. So far, their range of application is limited because of their comparably small size and low count rate. We present data on the development of superconducting tunnel junction (STJ) detector arrays to address both of these issues. A single STJ detector has a resolution of around $10 \mathrm{eV}$ below $1 \mathrm{keV}$ and can be operated at count rates of the order 10,000 counts/sec. We show that the simultaneous operation of several STJ detectors does not dimish their energy resolution significantly, and it increases the detector area and the maximum count rate by a factor given by the total number of independent channels.
\end{abstract}

Key words: cryogenic X-ray detectors, superconducting tunnel junctions, X-ray microcalorimeter, detector arrays

\section{INTRODUCTION}

Over the last 10 years, there has been increasing interest in energy-dispersive X-ray detectors operated at temperatures below $1 \mathrm{~K}$, which offer energy resolution comparable to wavelength-dispersive spectrometers (WDS) (Booth and Goldie, 1996; Twerenbold, 1996). The detector development was initially driven by X-ray and particle astrophysics (Kraus et al., 1989; McCammon et al., 1993). More recently, applications in microanalysis (Wollman et al., 1997), material science (Frank et al., 1998a), and biophysics (Benner et al., 1997; Friedrich et al., 1999) have emerged and are being pursued for scientific and commercial interest.

Received August 25, 1998; accepted October 15, 1998.

${ }^{\star}$ Corresponding author
Cryogenic X-ray detectors fall into two groups: microcalorimeters and superconducting tunnel junctions (STJs). Microcalorimeters measure the X-ray-induced temperature rise of a sensitive thermistor, typically a doped semiconductor (McCammon et al., 1993; Silver et al., 1996) or a superconducting transition edge sensor (Wollman et al., 1997). Microcalorimeters offer a very high-energy resolution below $8 \mathrm{eV}$ FWHM at $6 \mathrm{keV}$ (Wollman et al., 1997; Silver et al., 1996). This comes at the expense of a lower maximum count rate around 500 counts/sec, because the relaxation of thermal devices back to their equilibrium is intrinsically slow (Wollman et al., 1997). STJ detectors offer a slightly poorer resolution of $15.7 \mathrm{eV} \mathrm{FWHM}$ at $6 \mathrm{keV}$ (Verhoeve et al., 1998), although this may be improved if future devices employ superconductors with extremely low critical temperatures (Peacock et al., 1996; LeGrand et al., 
1998; Kraft et al., 1998). However, STJ detectors can be operated at significantly higher count rates of the order 10,000 counts/sec (Frank et al., 1998b).

One common challenge with cryogenic X-ray detectors is their comparably small size, typically $200 \times 200 \mu \mathrm{m}^{2}$. Simply increasing device size tends to degrade the resolution because of added heat capacity (for microcalorimeters) or capacitance (for STJs) or because of spatially varying detector response. Several approaches are being pursued to increase the effective detector area. One is to separate absorber and detector function and couple a large absorber with a small detector (Kraus et al., 1989; Ferger et al., 1996; Friedrich et al., 1997). This slows down the detector response and can complicate the spectral analysis. Another approach is to use a polycapillary focusing optics (Wollman et al., 1997) to increase the effective solid angle. This is at present rather costly and may exceed the detector's maximum count rate capability.

Here we report on the development of STJ detector arrays. Arrays increase not only the detector area but also the maximum count rate by a factor given by the total number of independent channels. The complexity and cost of operating a multi-channel array is acceptable in microanalysis and material science applications for arrays with 10 independent channels. Further increases in detector efficiency are then more likely to be obtained by using X-ray focusing optics.

\section{StJ Operating Principle}

Superconducting tunnel junctions consist of two superconducting electrodes separated by a thin insulator. They use the small energy gap by which excited single-particle states (so-called quasiparticles) are separated from the Cooper pairs that constitute the superconducting electronic ground state. An X-ray photon absorbed in one of the electrodes breaks Cooper pairs and thereby generates excess quasiparticles in proportion to its energy. The quasiparticles can tunnel through the insulating barrier and thereby produce an increased current proportional to the energy of the incoming X-ray. STJ detectors rely on measuring the excess current after X-ray absorption. As such, their operating principle is similar to that of conventional semiconductor $\mathrm{Si}(\mathrm{Li})$ or Ge detectors. One essential difference is that the gap in superconductors is of the order of $1 \mathrm{meV}$, a factor of about 1000 times smaller than in semiconductors. X-rays therefore generate roughly 1000 times more excess charge carriers in superconductors than in semiconductors. Theoretically, this results in an improved energy resolution by a factor of $\sqrt{1000} \approx 30$. For Nb-based STJs, the statistics of the initial charge generation ultimately limit the resolution to values between 0.8 and $1.7 \mathrm{eV}$ FWHM for X-ray energies between 0.2 and $1 \mathrm{keV}$ (Kraft et al., 1998). In most practical devices, additional fluctuations in the number of tunneling events reduce the theoretically attainable resolution in that energy range by a factor of $\approx 2.5$ (Mears et al., 1993).

Detector efficiency can be increased through a process called quasiparticle trapping (Kraus et al., 1989; Booth, 1987). It employs an absorber with a large energy gap in connection with a tunnel junction made from a smaller gap superconductor. Quasiparticles, which have diffused from the large gap absorber into the lower gap material, relax energetically by phonon emission and are then "trapped" in the potential well close to the tunneling barrier. Trapping separates the absorber from the detector function in the device. The absorber film can be made thick and from a high $\mathrm{Z}$ material for high efficiency, while the trapping layer can be made thin for fast tunneling. Trapping also increases the lifetime of the quasiparticles, as it keeps them away from the possibly degraded detector surface. If a large gap superconductor is used on either side of the tunnel junction, the quasiparticles will remain in the lower gap junction region until they recombine and form Cooper pairs again. These processes cause the current waveform from an STJ detector to exhibit a fast rise corresponding to fast quasiparticle diffusion to the tunnel barrier and a slower exponential decay with a time constant set by quasiparticle recombination. A cross-sectional drawing of our STJ detectors is shown in Figure 1.

The quasiparticle recombination time in the junction electrodes also determines the maximum count rate of STJ detectors. Ideally, quasiparticles from a previous absorption event should have relaxed to their ground state before the STJ detector measures another X-ray. Otherwise, pile-up will start to reduce the detector performance. Typical recombination times in our superconducting detectors are of the order of 2-10 $\mu \mathrm{sec}$ (Fig. 2). This allows STJ detector operation at count rates of several 1000 counts/sec with undiminished energy resolution. Operation at higher rates is possible with somewhat lower resolution due to pile-up (Frank et al., 1998b). 


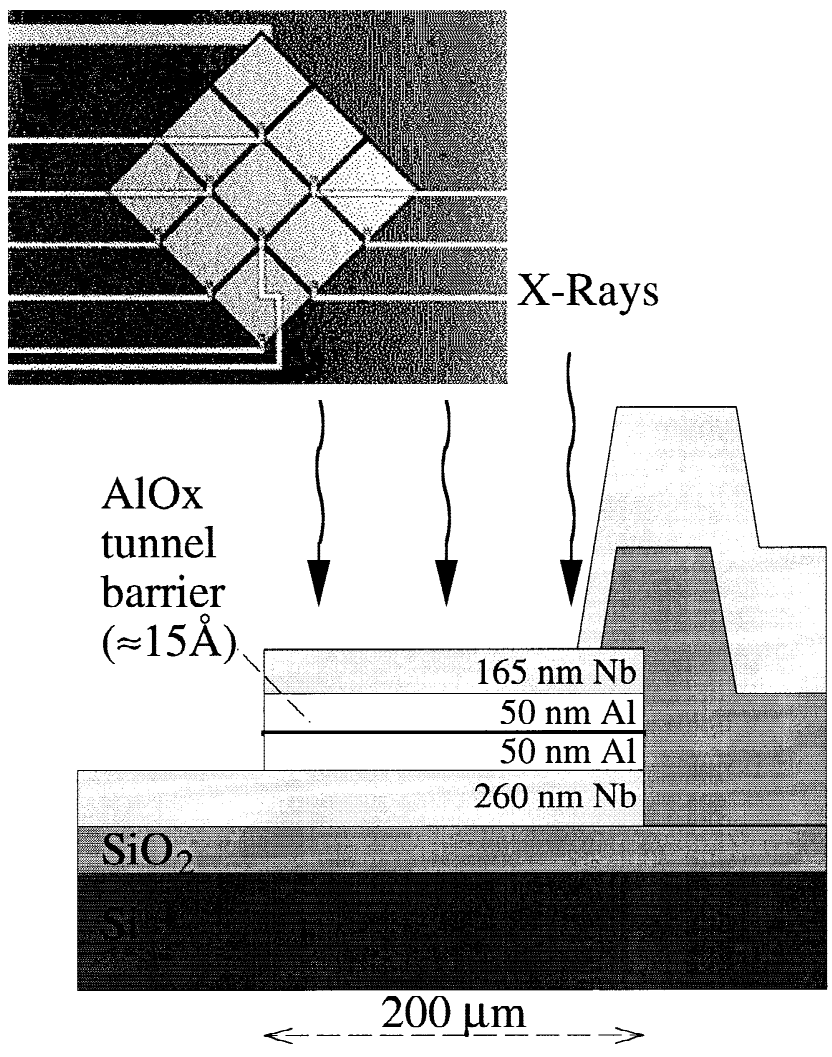

Figure 1. Cross section of an STJ detector. An X-ray breaks Cooper pairs in the top $\mathrm{Nb}$ absorber thereby generating free excess charges that scatter into the $\mathrm{Al}$ trap and produce a current pulse as they tunnel through the AlOx barrier. The inset shows a $3 \times 3$ array of $200 \times 200 \mu \mathrm{m}^{2}$ STJ detectors currently being developed.

\section{EXPERIMENTAL Results}

We are developing STJ spectrometers based on $\mathrm{Nb}-\mathrm{Al}-$ AlOx-Al-Nb thin-film technology. In earlier experiments, our small $50 \times 50 \mu \mathrm{m}^{2}$ devices showed an energy resolution between 4.6 and $8.9 \mathrm{eV}$ FWHM for X-ray energies between 0.2 and $1 \mathrm{keV}$ (LeGrand et al., 1998). The device discussed here is a linear array of four $200 \times 200 \mu \mathrm{m}^{2}$ STJ detectors. Each detector consists of a separate $265-\mathrm{nm}$ bottom $\mathrm{Nb}$ film, an Al-AlOx-Al tunnel junction with 50-nm thick $\mathrm{Al}$ electrodes, and a $165-\mathrm{nm}$ top $\mathrm{Nb}$ absorber. The devices are fabricated at Conductus, Inc. in Sunnyvale, CA, using a modified photolithographic trilayer process (Barfknecht et al., 1991). Details of the fabrication process have been published elsewhere (Mears et al., 1993).

The STJ detectors are operated in a liquid helium cryostat with an adiabatic demagnetization refrigerator (ADR) stage. It attains a base temperature of $60 \mathrm{mK}$ and can easily

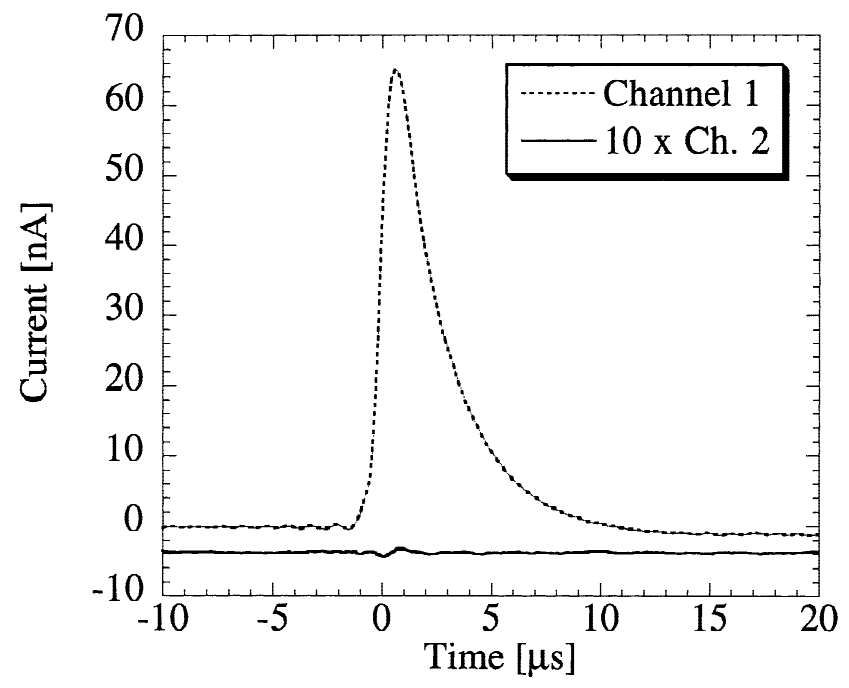

Figure 2. Average waveform of 1000 events, mostly $\mathrm{C} K$ and $\mathrm{O} K$ fluorescence. The trigger was set to channel 1 , and the signal induced through crosstalk in channel 2 is negligibly small. Channel 2 is magnified by a factor of 10 for clarity.

be attached to a synchrotron beam ring. The ADR has a hold time of 4-6 hr below $0.4 \mathrm{~K}$ and requires about $30 \mathrm{~min}$ to cycle. Note that the temperature does not need to be regulated for the operation of these devices as long as it remains below $0.4 \mathrm{~K}$. We apply a magnetic field of $\approx 100$ Gauss parallel to the device in the direction of the junction diagonal to suppress the $\mathrm{dc}$ Josephson current and reduce the magnitude of Fiske mode resonances (Fiske, 1964). This is required for bias stability at the operating voltage of $\sim 400$ $\mu \mathrm{V}$. For fluorescence experiments, we insert a sample stage through a load lock to within $5 \mathrm{~mm}$ of the STJ detector. A single $200 \times 200 \mu \mathrm{m}^{2}$ detector then covers a solid angle of $1.6 \cdot 10^{-3} \mathrm{sr}$. This small device size necessitates placing the sample close to the detector (and thus at cryogenic temperatures) and motivates the development of STJ arrays. Fortunately, photolithographic device fabrication and a preamplifier with an off-the-shelf field effect transistor (FET) input stage allow easy scaling to multi-element arrays. For details of the experimental setup see Frank et al. (1998a).

One concern for the operation of detector arrays is device uniformity. Our experience with photolithographically fabricated devices over the last 5 years has been that when a wafer contains high-quality junctions, all devices on that wafer tend to be good. The dc device I(V) characteristics tend to be very similar, although not identical. This is important because the same magnetic field must be used to suppress the dc Josephson current and the Fiske mode reso- 


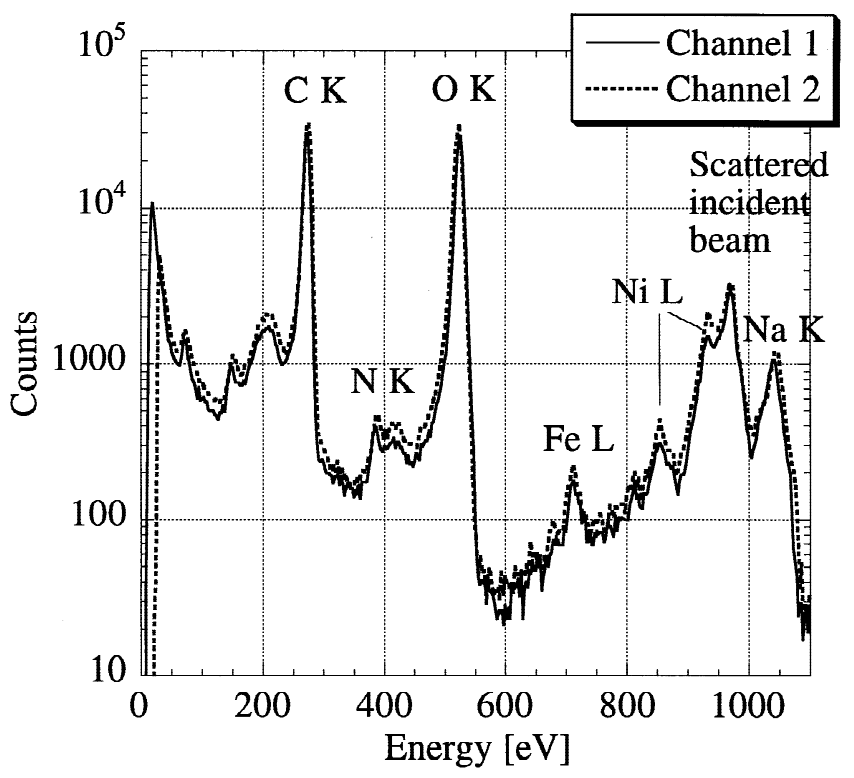

Figure 3. Fluorescence spectrum of the metalloprotein hydrogenase $(\sim 500 \mathrm{ppm} \mathrm{Ni})$ from two STJ detectors of a linear array. Both devices show a very similar spectral response. The excitation energy was $970 \mathrm{eV}$.

nances in all STJ devices of the array (den Hartogg et al., 1997). We found that it was not difficult to achieve the necessary suppression simultaneously for all the detectors in the array. Furthermore, future preamplifiers will employ dc voltage biasing rather than biasing at a constant $\mathrm{dc}$ current to further reduce the influence of Fiske mode resonances on device operation (Friedrich et al., 1997).

Figure 3 shows two fluorescence spectra of a hydrogenase sample, an enzyme from the bacterium Desulfovibrio gigas that is responsible for hydrogen evolution and uptake (Lancaster, 1988). The spectra were acquired simultaneously with two neighboring $200 \times 200 \mu \mathrm{m}^{2}$ detectors of a linear four-element array. Each of the detectors had its own electrical ground and electronic readout. Both detectors show nearly identical spectral response, differing only in an overall gain factor which has been corrected for by the calibration procedure used to convert the pulse height to $\mathrm{X}$-ray energy. The two prominent peaks at $277 \mathrm{eV}$ and 525 $\mathrm{eV}$ are $\mathrm{K}$-shell $\mathrm{X}$-rays from the carbon and oxygen that make up the bulk of the enzyme. The small peaks to the right of the oxygen line are the iron $L_{\alpha}$ and nickel $L_{\alpha}$ lines. The sample contains about $500 \mathrm{ppm} \mathrm{Ni}$ and 12 times more Fe. The number of counts in the Fe and the Ni lines do not reflect the 12:1 abundance ratio of $\mathrm{Fe}$ to $\mathrm{Ni}$ because $\mathrm{Fe}$ is excited less efficiently by the incident radiation, and residual gases frozen out on the sample attenuate the Fe signal more strongly than the higher-energy $\mathrm{Ni}$ fluorescence. The double peak structure above $900 \mathrm{eV}$ is mostly due to scatter of the incident $970 \mathrm{eV}$ X-ray beam, with a small shoulder on the low-energy side from the nickel $L_{\beta 3,4}$ line. The peak at $1041 \mathrm{eV}$ is due to sodium used in sample preparation which is excited by the second-order radiation of the synchrotron beam.

The resolution of the two devices individually is 10.7 and $10.9 \mathrm{eV}$ FWHM at carbon $K$ and 12.6 and $12.8 \mathrm{eV}$ at oxygen $K$, respectively. There is an uncertainty of $\pm 0.2 \mathrm{eV}$ in the resolution because of ambiguities as to how to subtract the spectral background at the different fluorescence lines. No electronic pulses were injected during this particular run to measure the contribution of the readout electronics to the line width. However, spectra taken earlier under identical conditions with the same device had an electronic noise contribution of around $9 \mathrm{eV}$. The response of the two devices is sufficiently similar to allow adding the two spectra. The combined spectrum of the two channels has a resolution of $10.9 \mathrm{eV}$ at carbon $\mathrm{K}(277 \mathrm{eV})$ and $12.9 \mathrm{eV}$ at oxygen $K(525 \mathrm{eV})$, respectively. Adding the two spectra broadens the lines only marginally. This makes us confident that scaling to larger arrays can be accomplished without much further loss in resolution.

While the detector resolution of the $200 \times 200 \mu \mathrm{m}^{2}$ devices is poorer than the resolution of smaller devices (LeGrand et al., 1998), it is still sufficient to fully separate the Ni $L$ and the Fe $L$ fluorescence from the strong oxygen $K$ line centered at $525 \mathrm{eV}$. In many applications, it is desirable to trade off resolution for larger solid-angle coverage as long as the detector can handle the higher count rate. This is particularly important for the fast analysis of dilute samples, where a sufficiently high number of counts in the weak signal line is accompanied by a huge number of counts from other elements in the sample. For example, the metalloprotein spectra shown in Figure 3 contain a total of $\approx 310,000$ counts just in the oxygen $K$ line, compared to $\approx 3200$ counts in the $\mathrm{Ni} L_{\alpha}$ line.

Crosstalk is another concern for operating multielement arrays. The question of whether an event in one of the detector elements will induce a signal in the neighboring detectors is particularly important when the two detectors are located immediately next to each other, as would be desirable in a detector array used with a focusing optics. We have measured the level of crosstalk between neighboring devices by triggering on events from only one channel and plotting the response of both channels averaged over 1000 individual events (Fig. 2). The signal in channel 1 is mostly 
due to carbon and oxygen $K$ fluorescence photons as seen in the spectra (Fig. 3). The waveform shows the fast rise time, limited by the response of the preamplifier, followed by an exponential decay with a time constant characteristic for the lifetime of the excess quasiparticles in the aluminum layers of the tunnel junction. The small correlated bipolar signal in channel 2 is the only evidence of crosstalk we have observed. It is magnified by a factor of 10 for clarity and it is not at all detectable without averaging. The amplitude of the signal in channel 2 is $<0.1 \%$ of the peak-to-peak signal in channel 1. Because of its short duration and bipolar nature, crosstalk effects are even further removed by the low-pass filtering procedure we typically use to reduce the effects of high-frequency noise. Crosstalk therefore does not limit the operation of our STJ detector arrays.

Future devices will replace the niobium with tantalum for better absorption efficiency at higher X-ray energies. We are also planning to build a $3 \times 3$ STJ array spectrometer (Fig. 1, inset), coupled with an X-ray focusing optics that offers a resolution below $10 \mathrm{eV}$ at energies up to $1 \mathrm{keV}$ and a total count rate well above 100,000 counts/sec. It will cover a solid angle of $0.1 \mathrm{sr}$ and will have a quantum efficiency between $10 \%$ and $20 \%$, depending on the particular type of optics used.

\section{SUMMARY}

We are developing Nb-based superconducting tunnel junctions (STJs) as high-resolution energy-dispresive X-ray spectrometers. Small $50 \times 50 \mu \mathrm{m}^{2}$ STJ detectors have an energy resolution of between 4.6 and $8.9 \mathrm{eV}$ FWHM for $\mathrm{X}$-ray energies between 0.2 and $1 \mathrm{keV}$ and can be operated at count rates of $\approx 10,000$ counts/sec. Larger $200 \times 200 \mu \mathrm{m}^{2}$ STJ detectors have somewhat poorer resolution, typically around $12 \mathrm{eV}$, due to higher device capacitance. We have operated two $200 \times 200 \mu^{2}$ devices from a multi-element array simultaneously without significant loss in detector resolution. A $3 \times 3$ detector array currently being developed will have an active area of $0.6 \times 0.6 \mathrm{~mm}^{2}$ and is expected to operate at total count rates of above 100,000 counts/sec.

\section{ACKNOWLEDGMENTS}

We thank Jan Batteaux and Jeff Moore for expert technical assistance. This work was performed under the auspices of the U.S. Department of Energy by Lawrence Livermore National Laboratory under contract W-7405-ENG-48 at the Stanford Synchrotron Radiation Laboratory (SSRL), which is operated by the Department of Energy, Office of Basic Energy Sciences. Funding for this research was provided by the Department of Energy, Office of Biological and Environmental Research, by the NIH through grant GM 44380, and by NASA through SBIR contract NAS5-32805 and through UV detector development grant NAG5-4137.

\section{REFERENCES}

Barfknecht AT, Ruby RC, Ko H (1991) A simple and robust Nb Josephson junction integrated circuit process. IEEE Trans Magn 27:3125-3128

Benner WH, Horn DM, Jaklevic JM, Frank M, Mears CA, Labov SE, Barfknecht AT (1997) Simultaneous measurement of flight time and energy of large matrix-assisted laser desorption ionization ions with a superconducting tunnel junction detector. $\mathrm{J} \mathrm{Am}$ Soc Mass Spectrom 8:1094-1102

Booth NE (1987) Quasiparticle trapping and the quasiparticle multiplier. Appl Phys Lett 50:293-295

Booth NE, Goldie DJ (1996) Superconducting particle detectors. Supercon Sci Technol 9:493-516

den Hartogg R, Peacock A, Verhoeve P, Jansen F, Verveer J, van Dordrecht A, Salmi J, Venn R (1997) On the development of a 3 $\times 3$ array of superconducting tunnel junctions for soft X-ray astronomy. SPIE Proc 3114:310-321

Ferger P, et al. (1996) Cryogenic particle detectors with superconducting phase transition thermometers. Nucl Instrum Methods A370:157-159

Fiske MD (1964) Temperature and magnetic field dependence of the Josephson tunneling current. Rev Mod Phys 36:221-222

Frank M, Mears CA, Labov SE, Hiller LJ, LeGrand JB, Lindeman MA, Netel H, Chow D, Barfknecht AT (1998a) Cryogenic highresolution X-ray spectrometers for SR-XRF and microanalysis. $J$ Synchrotron Rad 5:515-517

Frank M, Hiller LJ, LeGrand JB, Mears CA, Labov SE, Lindeman MA, Netel H, Chow D, Barfknecht AT (1998b) Energy resolution and high count rate performance of superconducting tunnel junction spectrometers. Rev Sci Instrum 69:25-31

Friedrich S, Segall K, Gaidis MC, Wilson CM, Prober DE, Kindlmann PJ, Szymkowiak AE, Moseley SH (1997) Single photon im- 
aging X-ray spectrometers using low-noise current preamplifiers with DC voltage bias. IEEE Trans Appl Supercon 7:3383-3386

Friedrich S, Hiller LJ, Frank M, le Grand JB, Mears CA, Nideroest B, Labov SE, Barfknecht AT, LeGros M, Cramer SP (1999) Superconducting high-resolution X-ray detectors for metalloprotein Ledge spectroscopy. J Electron Spectrosc Rel Phenom (in press)

Kraft S, Peacock A, Bavdaz M, Castello B, Collaudin B, Perez D, Venn R (1998) On the use of Hf-based superconducting tunnel junctions as high-resolution spectrometers for X-ray astronomy. SPIE Proc 3445:226-235

Kraus H, von Freilitzsch F, Jochum J, Mössbauer RL, Peterreins T, Proebst F (1989) Quasiparticle trapping in a superconductive detector system exhibiting high energy and position resolution. Phys Lett B231:195-202

Lancaster JR (ed) (1988) The Bioinorganic Chemistry of Nickel. New York: VCH Publishers

LeGrand JB, Mears CA, Hiller LJ, Frank M, Labov SE, Netel H, Chow D, Friedrich S, Lindeman MA, Barfknecht AT (1998) A superconducting tunnel junction detector with performance limited by statistical effects. Appl Phys Lett 73:1295-1297

McCammon D, Cui W, Juda M, Morgenthaler J, Zhang J, Kelley RL, Holt SS, Madejski GM, Moseley SH, Szymkowiak AE
(1993) Thermal calorimeters for high-resolution X-ray spectroscopy. Nucl Instrum Methods A326:157-165

Mears CA, Labov SE, Barfknecht AT (1993) Energy-resolving superconducting X-ray detectors with charge amplification due to multiple tunneling. Appl Phys Lett 63:2961-2963

Peacock A, Verhoeve P, Rando N, van Dordrecht A, Taylor BG, Erd C, Perryman MAC, Venn R, Howlett J, Goldie DJ, Lumley L, Wallis M (1996) Single optical photon detection with a superconducting tunnel junction. Nature 381:135-137

Silver E, LeGros M, Madden N, Beeman J, Haller E (1996) Highresolution, broad-band microcalorimeter for X-ray microanalysis. $X$-ray Spectrom 25:115-122

Twerenbold D (1996) Cryogenic particle detectors. Rep Prog Phys 59:349-426

Verhoeve P, Rando N, Peacock A, van Dordrecht A, Taylor BG, Goldie DJ (1998) High-resolution X-ray spectra measured using tantalum superconducting tunnel junctions. Appl Phys Lett 72: 3359-3361

Wollman DA, Irwin KD, Hilton GC, Dulcie LL, Newbury DE, Martinis JM (1997) High-resolution energy-dispersive microcalorimeter spectrometer for X-ray microanalysis. J Microsc 188:196223 